\title{
Injury-Induced Functional Plasticity in the Peripheral Gustatory System
}

\author{
Susan J. Hendricks, ${ }^{1}$ Suzanne I. Sollars, ${ }^{2}$ and David L. Hill ${ }^{3}$ \\ ${ }^{1}$ Bloedel Hearing Research Center, University of Washington, Seattle, Washington 98195, 2Department of Psychology, \\ University of Nebraska, Omaha, Nebraska 68182, and ${ }^{3}$ Department of Psychology, University of Virginia, Charlottesville, \\ Virginia 22904
}

Combining unilateral denervation of anterior tongue taste buds with a low-sodium diet in rats results in a rapid, dramatic, and selective attenuation of neurophysiological sodium taste responses from the intact side of the tongue. The transduction pathway responsible for the attenuated response is through the epithelial sodium channel (Hill and Phillips, 1994). Current experiments extend these findings by detailing the effects of experimentally induced injury on taste responses from anterior tongue taste receptors in sodium-restricted rats. Experiments focused on functional salt taste responses from the intact chorda tympani nerve in sodium-restricted rats in which a gustatory nerve was sectioned that innervates the anterior tongue (chorda tympani), the posterior tongue (glossopharyngeal), or palatal taste receptors (greater superficial petrosal) or in which a nongustatory nerve was sectioned that also has its target in the anterior tongue (trigeminal). An additional group was studied that received thermal injury to the anteroventral tongue. Substantial and selective suppression of sodium salt responses occurred in a graded manner generally related to the distance from the target field of the injury to anterior tongue taste buds. The order of effectiveness was: chorda tympani section $>$ trigeminal section $>$ thermal injury = glossopharyngeal section $>$ greater superficial petrosal section. These results support the hypothesis that local, diffusible factors liberated from immune-derived cells as a result of neural and/or epithelial damage are involved in regulating the transduction pathway responsible for sodium salt sensation, and that these factors may become evident through dietary sodium restriction.

Key words: taste; degeneration; ENaC; chorda tympani nerve; sodium restriction; immune; epithelia
The mature peripheral gustatory system is characterized as being inherently plastic. The primary reason for the plasticity is attributed to the normal turnover of taste receptor cells approximately every 10 d (Beidler and Smallman, 1965; Farbman, 1980; Hendricks et al., 2002). This presents a formidable challenge for gustatory neurons that have their receptive fields continually changing to maintain a constant afferent message transmitted to the brain. Coupled with the ongoing turnover of taste receptor cells, taste receptor cell structure and function are dependent on innervation (i.e., they are trophically dependent) (Zalewski, 1972; Cheal and Oakley, 1977). Loss of innervation results in a loss of the normal morphological appearance and responses of taste buds, whereas restoration of innervation results in a restoration of normal taste bud morphology and function (Cheal and Oakley, 1977; Cheal et al., 1977). Therefore, these bidirectional, plastic interactions between taste buds and the neurons that innervate them make the gustatory system an ideal model to examine the role of environmental effects and of degenerative/regenerative processes on sensory function and structure in mature animals.

Previous work (Hill and Phillips, 1994) has shown that the adult-regenerating gustatory system is especially susceptible to environmental manipulations. Unilateral chorda tympani nerve section in rats fed a sodium-restricted diet at adulthood results in a regenerated nerve that has attenuated responses specific to

Received April 26, 2002; revised July 23, 2002; accepted July 23, 2002.

This work was supported by National Institutes of Health Grants DC00407, DC04846, and HD07232.

Correspondence should be addressed to Dr. David L. Hill, Department of Psychology, P.O. Box 400400, University of Virginia, Charlottesville, VA 22904. E-mail: dh2t@virginia.edu.

Copyright (ㄷ) 2002 Society for Neuroscience $\quad 0270-6474 / 02 / 228607-07 \$ 15.00 / 0$ sodium salts. The transduction pathway primarily affected is through the epithelial sodium channel (ENaC) (Hill and Phillips, 1994). Therefore, environmental factors exert their effects on the function of ENaCs in receptor cells that newly form after a wholesale loss of previous generations. However, there are other, novel effects.

In the same rat, the contralateral, uncut nerve is supersensitive to sodium salts (Hill and Phillips, 1994). The supersensitivity does not occur immediately after sectioning the contralateral chorda tympani nerve. Rather, responses increase systematically after an initial subnormal response within $2 \mathrm{~d}$ after sectioning (i.e., $\sim 25 \%$ of controls). As found in the regenerated nerve, the neurophysiological alterations are selective and relate to functional changes in ENaCs. Moreover, the alterations in the intact nerve occur in the absence of reinnervation of the originally sectioned chorda tympani nerve (Hill and Phillips, 1994).

These large effects occur only under certain conditions. Chorda tympani sectioning must be accompanied by the sodiumrestricted diet to produce response alterations; the presence of only one of these conditions fails to produce functional changes. This points to important interactions between events that occur in response to nerve section and the physiological effects of maintenance on a low-sodium diet. Although the mechanism has not been identified, findings indicate that the immune system is involved in these processes (Phillips and Hill, 1996).

The current study is designed to define the injury-induced conditions that produce the functional alterations in this model system by focusing on sodium taste response alterations after axotomy of nerves innervating targets in the oral cavity or after localized thermal injury to the anteroventral tongue. Findings 
will provide a basis for determining the underlying molecular and cellular mechanisms.

\section{MATERIALS AND METHODS}

\section{Experimental manipulations}

All experiments were endorsed by the Animal Care and Use Committee of the University of Virginia and followed guidelines set by the National Institutes of Health and the Society for Neuroscience. Female Sprague Dawley rats (Harlan Sprague Dawley, Indianapolis, IN) were 40-45 d old at the time of surgery. Rats were initially injected with $0.1 \mathrm{cc}$ of atropine sulfate and subsequently anesthetized with methohexital sodium (50 mg/kg Brevital sodium, i.p.; Eli Lilly and Co., Indianapolis, IN) or ketamine/xylazine $(80 \mathrm{mg} / \mathrm{kg}$ ketamine; $3.33 \mathrm{mg} / \mathrm{kg}$ xylazine, i.p.). Rats were then placed into one of the following six conditions consisting of five types of nerve section and one group sustaining thermal injury. The term injury is used here to designate axotomy and the resultant loss of axons and target tissue, as well as tissue injury resulting from a localized burn to a nongustatory region of the anterior tongue. Figure 1 depicts the nerves sectioned and their respective targets.

Chorda tympani nerve section. The right chorda tympani was exposed in the neck of rats in this group $(n=6)$ and sectioned between the anterior belly of the digastric and masseter muscles where the chorda tympani nerve bifurcates from the lingual branch of the trigeminal. The lingual branch of the trigeminal nerve remained intact.

Lingual branch of the trigeminal nerve section. The right lingual branch of the trigeminal nerve was exposed as described for the chorda tympani surgery for this group of rats $(n=8)$. However, the lingual branch of the trigeminal nerve was sectioned proximal to the chorda tympani/lingual nerve juncture, whereas the chorda tympani nerve remained intact. Care was taken to leave the chorda tympani nerve undisturbed.

Glossopharyngeal nerve section. Via a single midline incision in the neck, both the right and left glossopharyngeal nerves were exposed and sectioned in this group $(n=8)$. Access to each glossopharyngeal nerve was gained by blunt dissection of muscle tissues at the level of the carotid notch. Briefly, the sternohyoid, omohyoid, and posterior belly of the digastric muscles were retracted to reveal the glossopharyngeal nerve. Care was taken not to damage the hypoglossal and superior laryngeal nerves. Bilateral glossopharyngeal nerve cuts were done to ensure degeneration of all circumvallate taste buds. Unilateral sectioning of the glossopharyngeal nerve was not done, unlike the other unilateral nervesectioned groups, because a single glossopharyngeal nerve can support 80-90\% of circumvallate taste buds (Whiteside, 1926; Oakley, 1970; State, 1977).

Greater superficial petrosal nerve section. An incision was made on the left, ventral portion of the neck in these rats $(n=8)$, and the posterior digastric muscle was retracted laterally to expose the tympanic bulla. A hole was placed in the ventral surface of the tympanic bulla, and the left greater superficial petrosal nerve was exposed immediately dorsal to the anterior portion of the tensor tympani muscle. The greater superficial petrosal nerve was sectioned, and the tensor tympani muscle and the digastric muscle were repositioned to their original location. Care was taken to avoid damage to the chorda tympani nerve as it courses through on the lateral edge of the tympanic bulla.

Unlike other gustatory nerves (i.e., chorda tympani and glossopharyngeal), each greater superficial petrosal nerve supplies taste buds on both sides of the palate, especially in the posterior palatine field (Fig. 1) (Cleaton-Jones, 1976; Miller and Spangler, 1982). Moreover, bilateral sectioning of this nerve fails to eliminate palatal taste buds (CleatonJones, 1976; Miller and Spangler, 1982). Thus, primarily because we could not achieve a complete loss of palatal taste buds even with bilateral sections, as is feasible with bilateral glossopharyngeal nerve section (see above), we chose to unilaterally section the greater superficial petrosal nerve on the side of the animal in which chorda tympani recordings were made (i.e., left side). This presents a contrasting condition compared with the other experimental groups in that neural degenerative processes will be shared with other nerve-sectioned groups but without wholesale target field degeneration. Uniquely, however, cell somata of the greater superficial petrosal nerve are contained within the geniculate ganglion along with somata of the chorda tympani nerve. Thus, there is a potential source of functional interaction in the ganglion between the two populations of cells after sectioning of the greater superficial petrosal nerve.

Thermal injury. An additional group of rats was studied to examine whether taste response alterations could be induced by localized injury to the tongue, instead of axotomy. Therefore, unlike previous groups, this

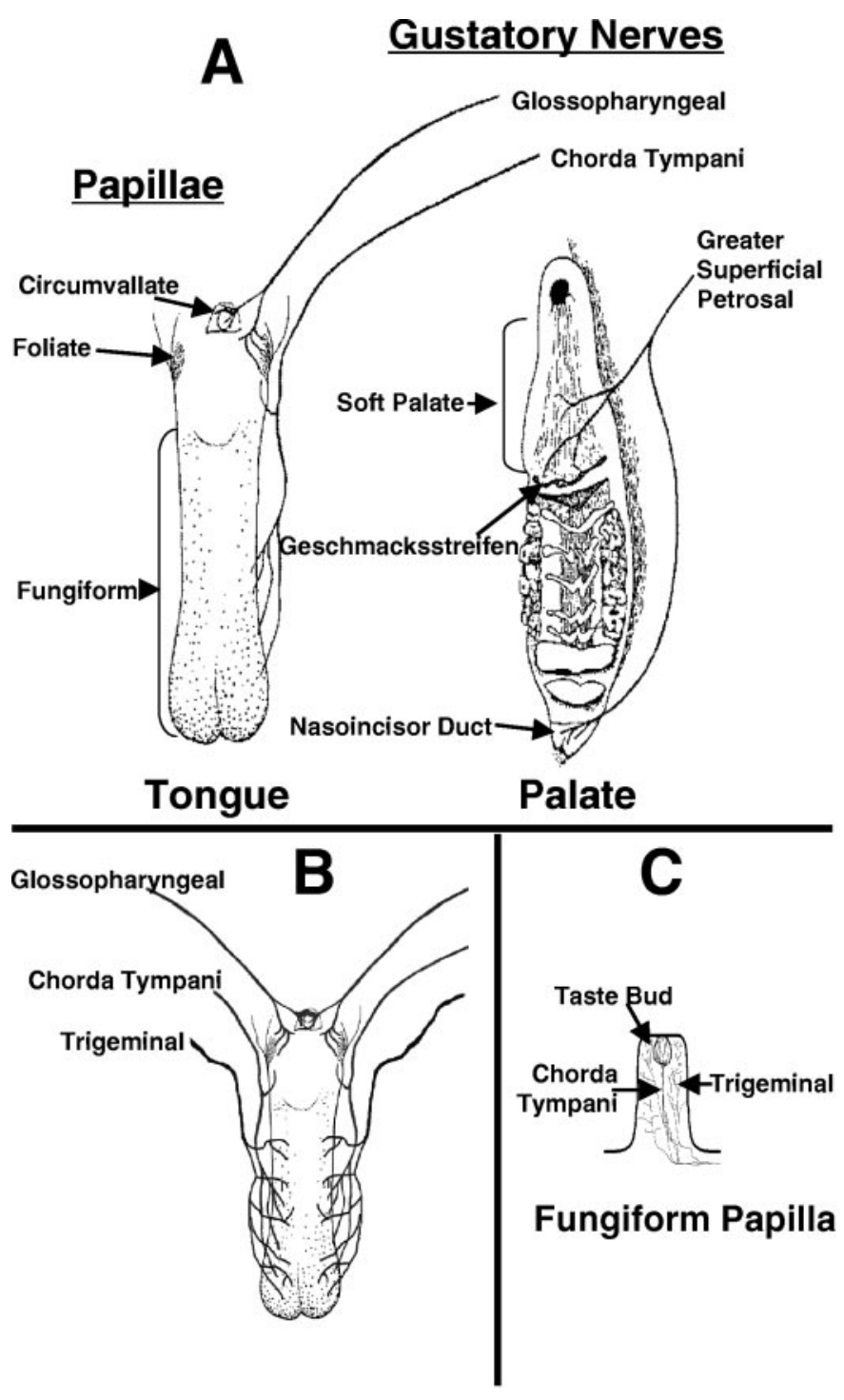

Figure 1. Drawings depicting the nerves sectioned and their respective targets. $A$, A diagram of the tongue (left) with the respective lingual papillae (Fungiform, Foliate, and Circumvallate) and the nerves that innervate taste buds in the papillae (Chorda tympani and Glossopharyngeal, respectively). Palatal taste buds (right) are contained within the nasoincisor duct, in the geschmacksstreifen, or in the soft palate. Palatal taste buds are innervated by the greater superficial petrosal nerve. Anterior is directed toward the bottom of the diagram. $B$, A diagram of gustatory nerves that innervate lingual taste buds (Chorda tympani and Glossopharyngeal) and the trigeminal nerve with its respective field of innervation. $C$, A diagram of a coronal section through a fungiform papilla showing the location of taste buds and the course of the chorda tympani and trigeminal nerves (up is dorsal).

group did not sustain nerve section. Instead, rats $(n=7)$ sustained thermal injury to the left, anteroventral tongue. Specifically, the tongue was gently pulled from the mouth, and the ventral surface was exposed. A low-heat cautery tool (Roboz Surgical, Gaithersburg, MD) was applied to the ventral tongue surface unilaterally, $\sim 3 \mathrm{~mm}$ from the tip for $1-2 \mathrm{sec}$ to induce a small thermal injury $\left(6 \mathrm{~mm}^{2}\right)$. Damage was limited to the most superficial layers, as evidenced by a white discoloration, and care was taken to avoid interruption of large blood vessels, taste buds on the ventral tongue, and the dorsal lingual epithelia. There was little evidence of damage by the time of neurophysiological recordings.

Sham-operated controls. To compare results with previous work that examined chorda tympani function after gustatory nerve section (Hill and Phillips, 1994), we chose to compare results from rats with nerve 
section to rats that received sham chorda tympani surgeries. Specifically, sham-operated rats $(n=5)$ were surgically prepared as described for the chorda tympani and trigeminal-sectioned groups, with the exception of sustaining a nerve section. To examine surgical influences not related to nerve sectioning with other surgical approaches, an additional two groups of sham-operated controls [sham-operated greater superficial petrosal nerve $(n=2)$ and sham-operated glossopharyngeal nerve $(n=$ 2)] were also prepared.

Rats receiving nerve section or sham-operated control procedures had wounds closed with suture thread followed by topical application of betadine and recovered from anesthesia on a heating pad. All rats received two injections of furosemide $(10 \mathrm{mg}$, i.p., over $24 \mathrm{hr})$ and were fed low-sodium chow $(0.03 \% \mathrm{NaCl}$; ICN Biochemicals, Costa Mesa, CA) and distilled water ad libitum.

\section{Neurophysiology}

Animals were deeply anesthetized with sodium pentobarbital $(50 \mathrm{mg} / \mathrm{kg}$ Nembutal, i.p.) 4-12 d after the initial surgery. This is the period during which chorda tympani responses are low after sectioning of the contralateral chorda tympani nerve (Hill and Phillips, 1994). The animals were tracheotomized and placed on a circulating water heating pad to maintain body temperature. Hypoglossal nerves were transected bilaterally to prevent tongue movement, and the animal was placed in a nontraumatic head holder. The left chorda tympani nerve was isolated using a mandibular approach (Hill and Phillips, 1994). The nerve was exposed near the tympani bulla, cut, desheathed, and placed on a platinum electrode. A second electrode was placed in nearby muscle to serve as ground. A mixture of petroleum jelly and mineral oil was placed in the cavity around the nerve. Whole chorda tympani neural activity was fed to a Grass Instruments (Quincy, MA) low impedance input stage amplifier. The filtered signal was monitored with an audio loudspeaker and oscilloscope. The signal was integrated (time constant, $0.4-0.6 \mathrm{sec}$ ) and fed to a strip chart recorder (Linseis, Princeton Junction, NJ) for later analysis of response magnitudes.

\section{Stimulation procedure}

All chemicals were reagent grade and prepared in distilled water. Neural responses to a concentration series of $0.05,0.1,0.25$, and $0.5 \mathrm{M} \mathrm{NaCl}$, sodium acetate $(\mathrm{NaAc})$, and $\mathrm{KCl}$ were recorded. Each concentration series was bracketed by applications of $0.5 \mathrm{M} \mathrm{NH}_{4} \mathrm{Cl}$. In addition, responses were recorded to the nonsalt stimuli of $0.01 \mathrm{~N} \mathrm{HCl}, 0.01 \mathrm{M}$ quinine hydrochloride, and $1 \mathrm{~m}$ sucrose. Solutions were applied to the tongue in $5 \mathrm{ml}$ aliquots with a syringe and allowed to remain on the tongue for $\sim 40 \mathrm{sec}$. After each solution application, the tongue was rinsed with distilled water for $\geq 1 \mathrm{~min}$. Chorda tympani responses were calculated as follows: the height of the tonic response was measured 20 sec after application, and response magnitudes were expressed as ratios relative to the mean of $0.5 \mathrm{M} \mathrm{NH}_{4} \mathrm{Cl}$ responses before and after stimulation (Hill and Phillips, 1994). Response data were retained for analysis only when $0.5 \mathrm{M} \mathrm{NH}_{4} \mathrm{Cl}$ responses that bracketed a concentration series varied by $<10 \%$.

\section{Data analysis}

Response data were grouped according to surgery condition and expressed as mean \pm SEM. Initially, we were interested in comparing experimental groups with sham-operated chorda tympani nerve controls. ANOVAs followed by Dunnett's tests $(p<0.05)$ were used to detect differences between each surgical condition and sham-operated controls for each stimulus concentration. Therefore, sham-operated control responses were used as the standard to which all others were compared. Because initial reports (Hill and Phillips, 1994; Phillips and Hill, 1996) demonstrated significant suppression in sodium responses after contralateral chorda tympani nerve section, we were also interested in testing whether experimental groups differed from rats with chorda tympani sections. Accordingly, statistical procedures were done using the chorda tympani-sectioned animals as the comparison group. Significant probability levels for the Dunnett's tests are reported in Results.

\section{RESULTS}

\section{Salt responses}

Responses from sham-operated control rats

Responses to concentration series of $\mathrm{NaCl}, \mathrm{NaAc}$, and $\mathrm{KCl}$ for chorda tympani/trigeminal sham-operated controls were similar

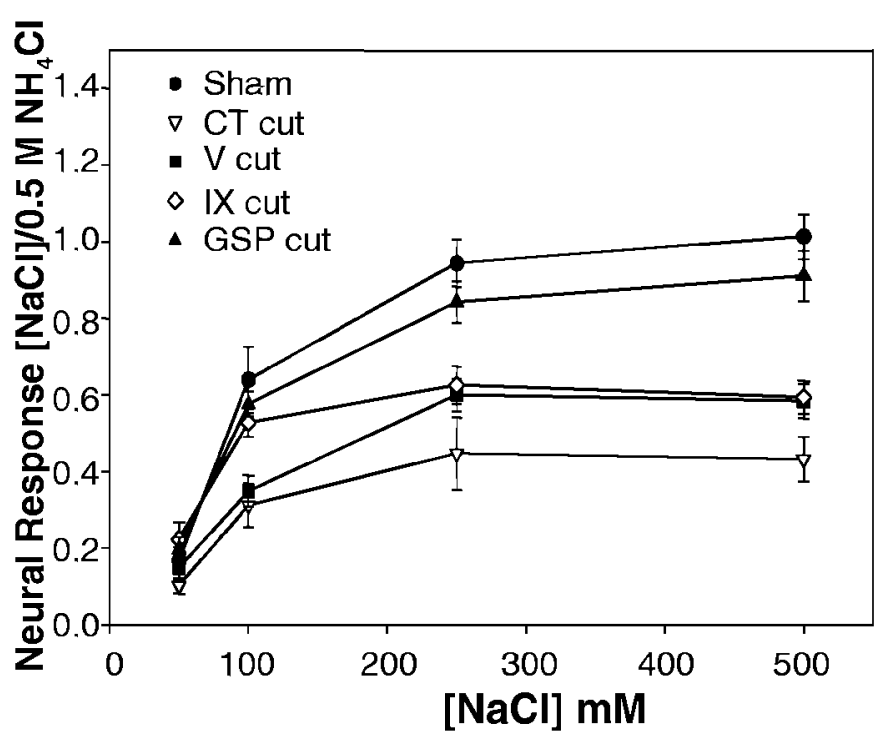

Figure 2. Mean \pm SEM response-concentration functions from the uncut chorda tympani nerve for $\mathrm{NaCl}$ in sodium-restricted rats with a sham nerve cut (Sham), unilateral chorda tympani cut (CT cut), unilateral trigeminal nerve cut (Vcut), bilateral glossopharyngeal nerve cut (IX cut), or unilateral greater superficial petrosal nerve cut (GSP cut). Axotomy of the chorda tympani, trigeminal, or glossopharyngeal nerves attenuates relative response magnitudes from the intact chorda tympani nerve to $\mathrm{NaCl}$. Responses in rats with the greater superficial petrosal nerve cut were similar to sham-operated rats.

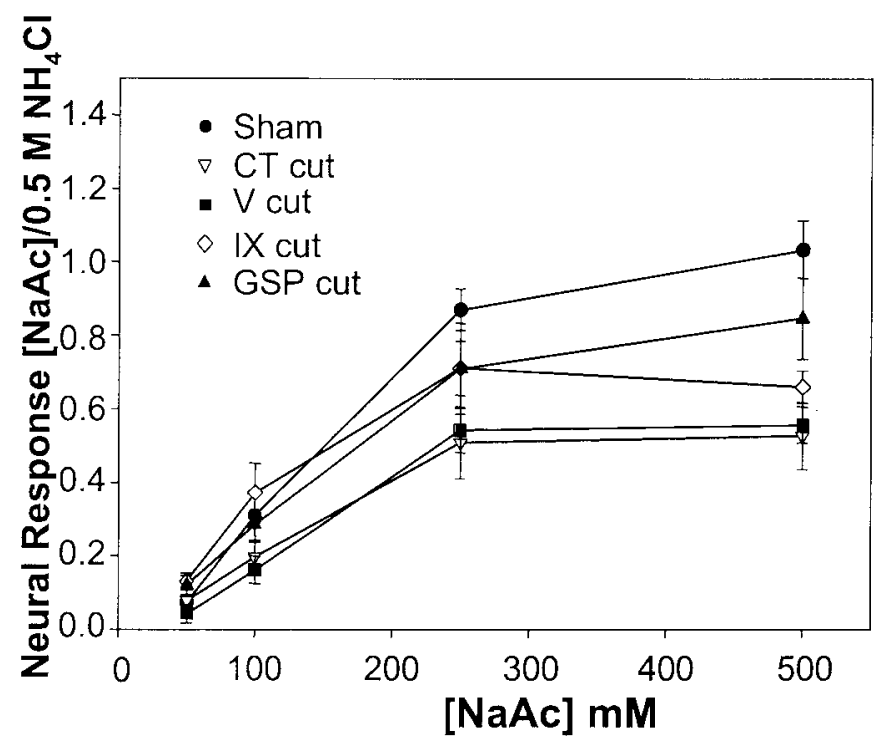

Figure 3. Mean \pm SEM response-concentration functions from the uncut chorda tympani nerve for $\mathrm{NaAc}$ in sodium-restricted rats with a sham nerve cut (Sham), unilateral chorda tympani cut (CT cut), unilateral trigeminal nerve cut (V cut), bilateral glossopharyngeal nerve cut (IX cut), or unilateral greater superficial petrosal nerve cut (GSP cut). Response attenuation at the highest concentrations occurred in rats receiving a nerve section, except in those rats sustaining damage to the greater superficial petrosal nerve.

to control data reported by Hill and Phillips (1994). As stimulus concentration increased, relative response magnitudes also increased (Figs. 2, 3, and 4). Although statistical comparisons were not made between the other sham-operated animals (i.e., greater superficial petrosal nerve controls and glossopharyngeal nerve 


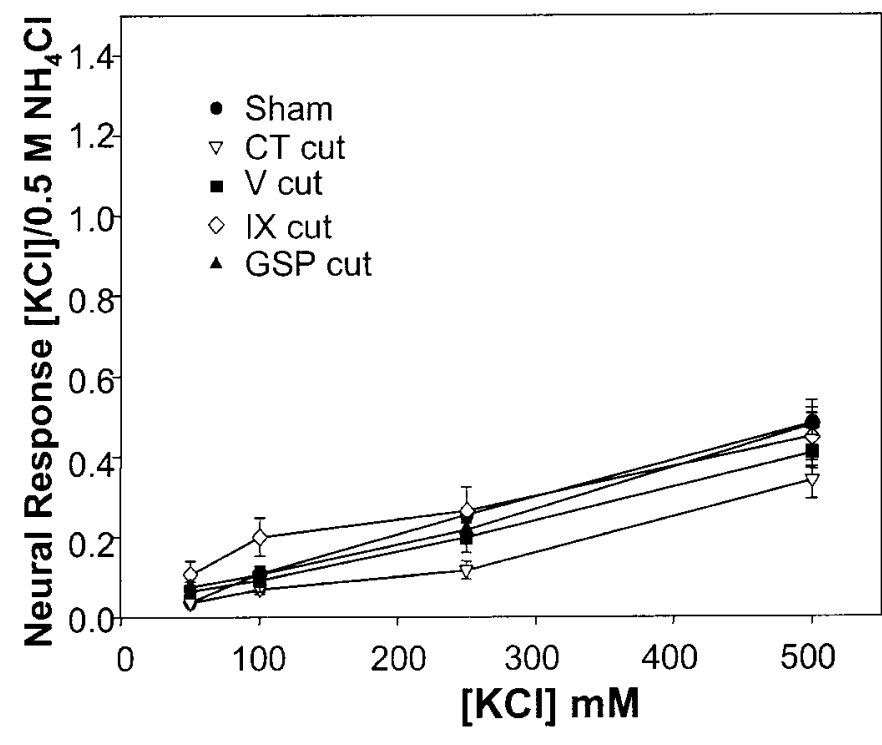

Figure 4. Mean \pm SEM response-concentration functions from the uncut chorda tympani nerve for $\mathrm{KCl}$ in sodium-restricted rats with a sham nerve cut (Sham), unilateral chorda tympani cut (CT cut), unilateral trigeminal nerve cut ( $V c u t)$, bilateral glossopharyngeal nerve cut (IX cut), or unilateral greater superficial petrosal nerve cut $(G S P$ cut $)$. Responses were similar among groups to each concentration of $\mathrm{KCl}$.

controls) and chorda tympani/trigeminal controls, all responses were within the $95 \%$ confidence intervals of the respective chorda tympani/trigeminal control means. Therefore, we found no evidence that sham surgery had an influence on chorda tympani function, although rats were placed on a NaCl-restricted diet. Data were not pooled among control groups; only chorda tympani/trigeminal control means were used for statistical analyses.

\section{Responses from the uncut chorda tympani nerve in unilateral} chorda tympani nerve-sectioned rats

As reported by Hill and Phillips (1994), there were dramatic and specific alterations in taste responses in the intact chorda tympani nerve in rats sustaining unilateral chorda tympani nerve section coupled with dietary $\mathrm{NaCl}$ restriction. Responses to $0.1,0.25$, and $0.5 \mathrm{M} \mathrm{NaCl}$ were $\sim 50-60 \%$ lower than in controls (Figs. 2 and 5) ( $p=0.001-0.0001)$, and responses to 0.25 and $0.5 \mathrm{~m} \mathrm{NaAc}$ were $40-50 \%$ lower than controls (Fig. 3) ( $p=0.019$ and 0.0001 , respectively). The effect on chorda tympani responses is as least as much as reported previously (Hill and Phillips, 1994; Phillips and Hill, 1996) when unilateral chorda tympani section was coupled with dietary $\mathrm{NaCl}$ restriction. No differences were found for $\mathrm{KCl}$ responses (Fig. 4).

Responses from the chorda tympani nerve in unilateral lingual branch of the trigeminal nerve-sectioned rats

Similar to chorda tympani-sectioned rats, unilateral section of the lingual branch of the trigeminal nerve resulted in reduced relative responses to $0.1,0.25$, and $0.5 \mathrm{M} \mathrm{NaCl}$ (Figs. 2 and 5) $(p=$ $0.001-0.0001)$ and to $0.5 \mathrm{M} \mathrm{NaAc}$ (Fig. 3) ( $p=0.0001)$. Responses to $\mathrm{NaCl}$ were reduced $\sim 40-50 \%$ to $\mathrm{NaCl}$ and by $40-$ $45 \%$ to $\mathrm{NaAc}$. No differences in responses to $\mathrm{KCl}$ were seen between trigeminal nerve-sectioned rats and controls (Fig. 4). Furthermore, no significant differences in responses were found between this group and sodium-restricted rats that had sustained unilateral chorda tympani section (Figs. 2-4).

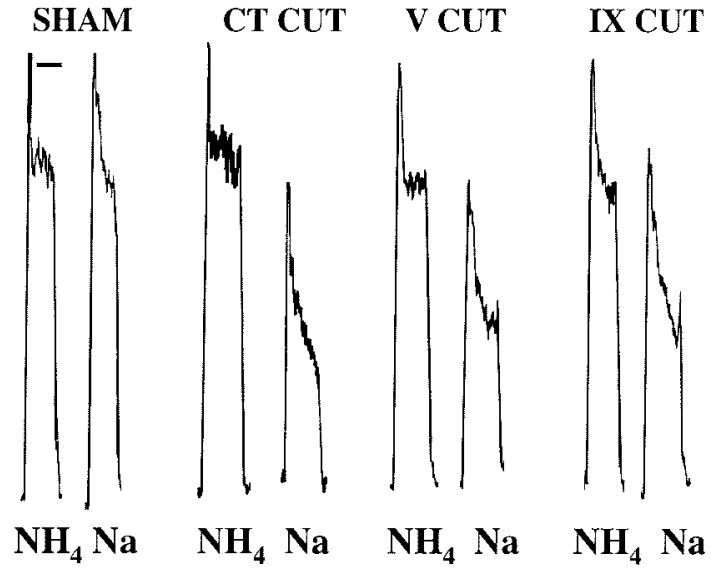

Figure 5. Integrated responses from the uncut chorda tympani nerve to $0.5 \mathrm{M} \mathrm{NH}_{4} \mathrm{Cl}\left(\mathrm{NH}_{4}\right)$ and $0.5 \mathrm{M} \mathrm{NaCl}(\mathrm{Na})$ in sodium-restricted rats with a sham operation (SHAM), unilateral chorda tympani cut (CT CUT), unilateral trigeminal nerve cut $(V C U T)$, or bilateral glossopharyngeal nerve cut (IX CUT). Steady-state responses (20 sec after stimulus onset) in sham rats were similar to $\mathrm{NH}_{4} \mathrm{Cl}$ and $\mathrm{NaCl}$, whereas the response to $\mathrm{NaCl}$ was much less than the response to $\mathrm{NH}_{4} \mathrm{Cl}$ in all other groups. Responses from rats receiving thermal injury are not shown but are similar to glossopharyngeal nerve cut rats.

\section{Responses from the chorda tympani nerve in bilateral glossopharyngeal nerve-sectioned rats}

Compared with chorda tympani/trigeminal sham-operated controls, rats sustaining bilateral glossopharyngeal nerve section had suppressed responses of $\sim 35-40 \%$ to 0.25 and $0.5 \mathrm{M} \mathrm{NaCl}$ (Figs. 2 and 5) $(p=0.0001)$ and to $0.5 \mathrm{M} \mathrm{NaAc}$ (Fig. 3$)(p=0.004)$. No differences between groups were found in $\mathrm{KCl}$ responses (Fig. 4).

Compared with responses from the intact nerve in rats sustaining chorda tympani section coupled with the low-sodium diet, only the response to $0.1 \mathrm{M} \mathrm{NaCl}$ was significantly different (Fig. 2) $(p=0.002)$.

Responses from the chorda tympani nerve in unilateral greater superficial petrosal nerve-sectioned rats

All mean relative responses to the concentration series of $\mathrm{NaCl}$, $\mathrm{NaAc}$, and $\mathrm{KCl}$ were similar to controls. However, compared with chorda tympani-sectioned rats, responses to $0.1,0.25$, and $0.5 \mathrm{M}$ $\mathrm{NaCl}$ were significantly greater in this group (Figs. $2-4)(p=$ 0.02-0.0001).

\section{Responses from the chorda tympani nerve in rats sustaining thermal injury}

As shown in Figure 6, the thermal injury produced response suppression to sodium salts intermediate between sham-operated controls and sodium-restricted rats with unilateral chorda tympani section. Indeed, the thermal-induced effect was similar to that produced by sectioning the trigeminal nerve. Responses to $0.1,0.25$, and $0.5 \mathrm{M} \mathrm{NaCl}$ and to $0.5 \mathrm{~m} \mathrm{NaAc}$ were significantly lower than in controls (Fig. 6) ( $\mathrm{NaCl}, p=0.02$ and 0.0001 ; $\mathrm{NaAc}$, $p=0.03$ ). Responses to $0.5 \mathrm{M} \mathrm{NaCl}$ were significantly greater than chorda tympani-sectioned rats fed the low-sodium diet $(p=$ 0.04). Therefore, localized injury to the ventral tongue epithelium produced specific alterations in taste function from a nearby gustatory nerve.

\section{Nonsalt responses}

Comparisons between controls and each of the five surgical groups for responses to $0.01 \mathrm{~N} \mathrm{HCl,} 0.01 \mathrm{M}$ quinine hydrochloride, 

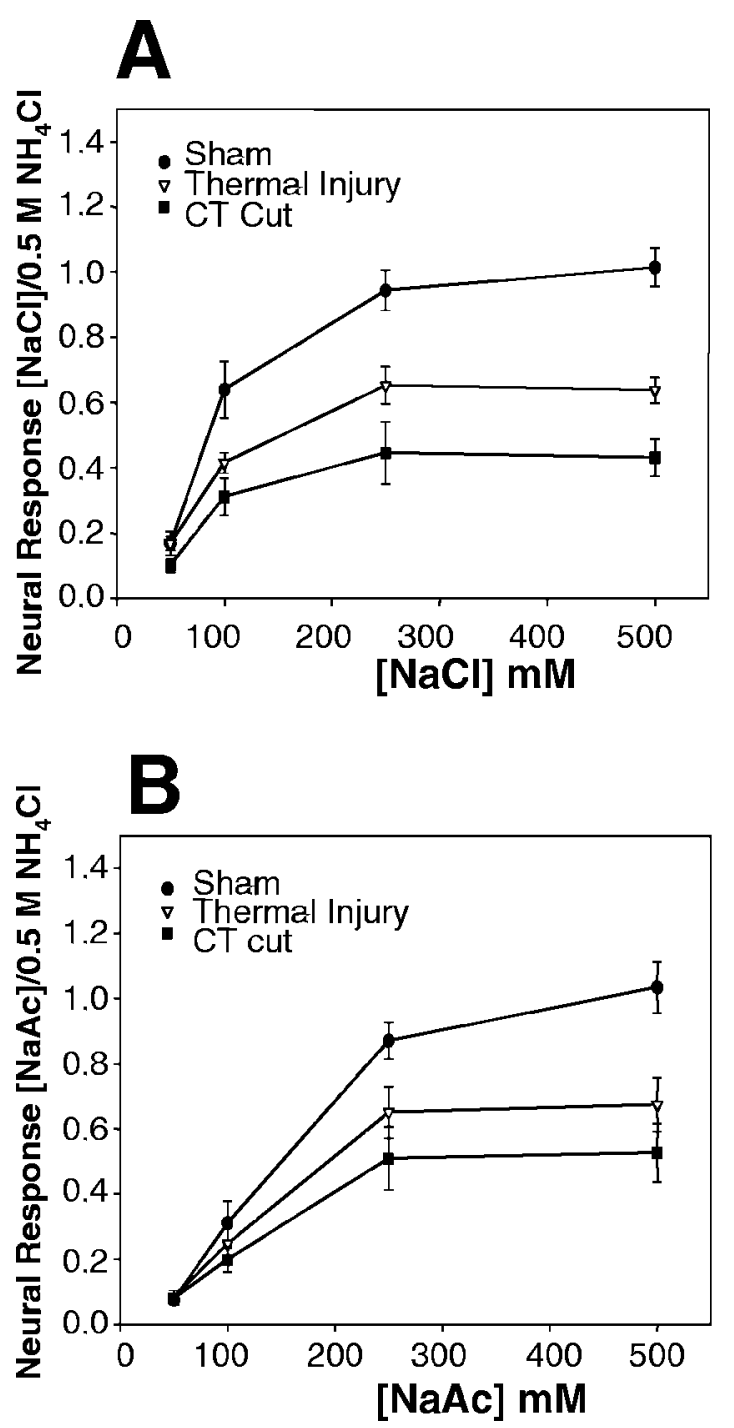

Figure 6. Mean \pm SEM response-concentration functions from the chorda tympani nerve for $\mathrm{NaCl}(A)$ and $\mathrm{NaAc}(B)$ in sodium-restricted rats with a sham nerve cut (Sham) or unilateral chorda tympani cut $(C T$ $C u t)$ or in rats that sustained thermal injury (Thermal Injury) to the anteroventral tongue. The thermal injury produced response suppression to sodium salts intermediate between sham and chorda tympani cut rats.

and $1 \mathrm{~m}$ sucrose revealed that there were no significant differences (Fig. 7). Response magnitudes from the chorda tympani nerve were small to each of the three nonsalt stimuli, regardless of the experimental group.

\section{DISCUSSION}

The findings reported here demonstrate that the adult peripheral gustatory system is functionally plastic when dietary sodium restriction is combined with sectioning a nerve that has its target in the tongue or with localized thermal injury to the tongue. Not only are sodium taste responses suppressed in the chorda tympani nerve within days after sectioning of the contralateral chorda tympani in sodium-restricted rats, but injury to other nerves (and targets) in the tongue also suppresses sodium salt responses from the chorda tympani nerve. Furthermore, the nerve sectioned does not have to be a gustatory nerve. Sectioning the lingual branch of the trigeminal nerve, which innervates nongustatory targets in the tongue, produced effects similar to that of chorda tympani sec-

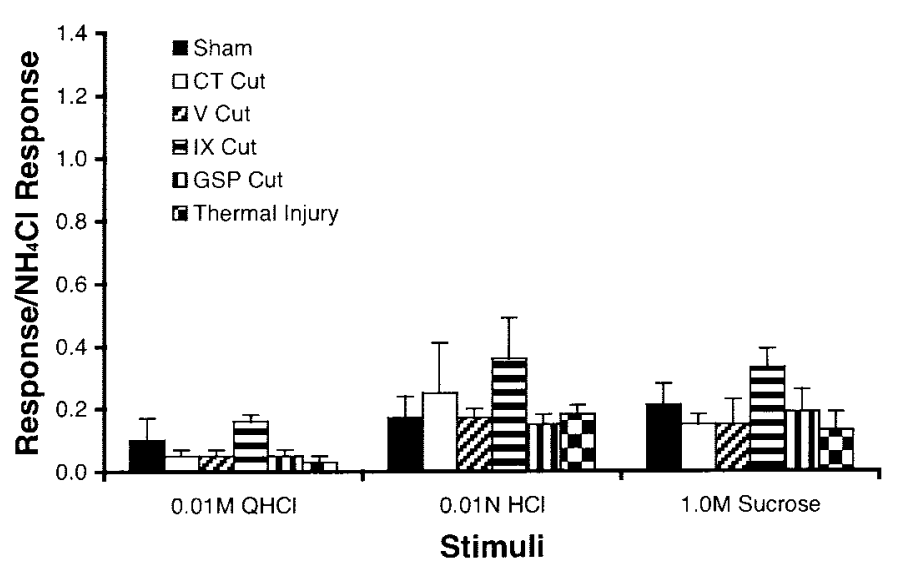

Figure 7. Mean $\pm \mathrm{SEM}$ relative responses from the uncut chorda tympani nerve to $0.01 \mathrm{M}$ quinine hydrochloride $(\mathrm{QHCl}), 0.01 \mathrm{~N} \mathrm{HCl}$, and $1 \mathrm{M}$ sucrose in sodium-restricted rats with a sham nerve cut (Sham), unilateral chorda tympani cut (CT Cut), unilateral trigeminal nerve cut $(V C u t)$, bilateral glossopharyngeal nerve cut (IX Cut), unilateral greater superficial petrosal nerve cut (GSP Cut), or thermal injury (Thermal Injury). Responses were similar among groups to each nonsalt stimulus.

tioning. Conversely, sectioning a gustatory nerve in sodiumrestricted rats that does not innervate lingual taste buds had no effect on chorda tympani function. Sodium-restricted rats that received sections of the greater superficial petrosal nerve unilateral to the chorda tympani nerve from which recordings were made had taste responses similar to sham-operated controls.

From our results with rats sustaining localized thermal injury to the tongue, it is apparent that nerve section and subsequent receptor cell degeneration are not a requirement for sodium response attenuation in sodium-restricted rats. Localized injury to the tongue that presumably produces an inflammatory response also affects sodium taste responses when combined with dietary sodium restriction.

It is apparent that the large attenuation in sodium salt responses (i.e., as much as 60\%) must be the result of coupling nerve section along with the dietary manipulation. Nerve section or dietary sodium restriction alone fails to produce response changes. For example, our sham-operated controls were maintained on the sodium-restricted diet, and their responses were similar to those reported for other control groups (also see Hill and Phillips, 1994). From previous work (Hill and Phillips, 1994), it is also evident that unilateral sectioning of the chorda tympani nerve without the dietary manipulation (i.e., nerve section controls) fails to attenuate sodium salt taste responses. The current findings extend these results by showing that even combining transection of a gustatory nerve that innervates taste buds not located on the tongue (i.e., the greater superficial petrosal nerve) with dietary manipulations fails to affect sodium salt taste responses in the chorda tympani nerve. Therefore, the effects reported here show that the attenuation in sodium salt response can be generalized to interruption of gustatory and nongustatory nerves with their targets in the tongue (Hill and Phillips, 1994) but cannot be generalized to gustatory nerves that innervate palatal taste buds. Moreover, the concentration-dependent taste response effects shown here are similar to experimentally induced effects demonstrated previously in unilateral chorda tympanisectioned rats (Hill and Phillips, 1994; Phillips and Hill, 1996). This suggests that only ENaCs recruited for sodium transduction at relatively high concentrations are affected by experimental 
manipulations, presumably by decreasing the number of functional channels.

Perhaps the most intriguing finding from these experiments is that the magnitude of the effect is related to the distance of the target field innervated by the nerve cut from taste receptors innervated by the chorda tympani nerve from which responses were recorded. Specifically, the order of effectiveness of injury was: chorda tympani section $>$ trigeminal section $>$ glossopharyngeal sections $>$ greater superficial petrosal section $=$ shamoperated controls. The only relatively minor exception to this is that the target field of the sectioned chorda tympani nerve is approximately the same distance as the trigeminal nerve from the intact taste receptors (i.e., the contralateral chorda tympani); yet chorda tympani sections created the largest effect. It should be noted that no difference in results occurred if the trigeminal nerve ipsilateral or contralateral to the recorded chorda tympani nerve was sectioned (data not shown). It is possible that the loss of taste receptor cells in addition to neural degeneration attendant to chorda tympani nerve section and not trigeminal nerve section may account for this difference. That is, degenerative cellular processes induced by chorda tympani section may be more effective qualitatively and quantitatively in altering taste responses in intact taste buds compared with processes resulting from trigeminal nerve section. Although greater superficial petrosal nerve sections produce neural degeneration and, presumably, immunerelated responses, it should be noted that there may be relatively little taste bud degeneration (Cleaton-Jones, 1976; Miller and Spangler, 1982). As such, the degree of degeneration and/or distance from fungiform taste receptors may be responsible for the lack of attenuated sodium taste responses. Finally, the site of cellular damage in the thermal injury group may be a similar distance from taste receptors innervated by the chorda tympani nerve from which responses were recorded as the target field of the trigeminal nerve. However, unlike the nerve-sectioned groups, injury was incurred by epithelial cells and few (if any) neural processes. Therefore, an effect that is slightly less pronounced than that seen with trigeminal section would be expected.

One explanation for the effects seen here and previously (Hill and Phillips, 1994) is that efferent systems carried through the chorda tympani nerve are disrupted. This seems unlikely, because a diverse type and number of nerves in the tongue can be sectioned with graded effects on chorda tympani function. Indeed, there is little evidence of a significant efferent taste pathway by way of gustatory nerves (Farbman and Hellekant, 1978). If anything, sectioning the ipsilateral greater superficial petrosal nerve should have had a significant influence on chorda tympani function if the effects were exclusively neurally mediated because of the close anatomical relationship within the geniculate ganglion.

An alternative explanation favored here is that nerve sectioninduced factors (e.g., cytokines) are produced in response to inflammation and/or phagocytic activity. Indeed, there is evidence in the adult rat visual system that a unilateral section of one optic nerve has immune-related effects on the contralateral retinofugal system (Bodeutsch et al., 1998). In the gustatory system, these putative immune-derived factors could act on distant taste receptors to alter sodium salt taste transduction pathways. This is consistent with the finding that injury to tissue closest to the functional taste receptors (e.g., trigeminal nerve) would have a greater effect than injury to those that are more distant (e.g., glossopharyngeal nerve). It appears, therefore, that the effects shown here are nonspecific with regard to the type of injury sustained. Rather, it is the location and amount of injury that may determine alterations in sodium taste function.

However, a problem remains. That is, why must the rats be fed a low-sodium diet to express the injury-induced effects? In response to injury, the immune system is activated to clear cellular debris. For both axotomy- and thermal-induced injury, immune cells (e.g., leukocytes) phagocytize debris and, importantly, release a number of cellular products, including a variety of cytokines, neurotrophins, and growth factors (for review, see Abbas et al., 2000). Once released from immune cells, soluble products have access not only to neighboring but also to distant sites (i.e., intact taste buds) by way of the circulatory and lymphatic systems in the dorsal tongue epithelium (Hellekant, 1976; Maher, 1985). Of interest to this study, immune cells and their products can influence normal and injured neuroepithelial cells (Lu and Richardson, 1991; Jones and Corwin, 1993, 1996; Popovich et al., 1996; Warchol, 1997; Bhave et al., 1998). It is also clear that phagocytic cells appear in circumvallate and foliate taste buds after glossopharyngeal sections (Suzuki et al., 1996, 1997). These processes would be expected to occur in both $\mathrm{NaCl}$-replete and $\mathrm{NaCl}$-restricted rats. To be consistent with the current data, injury-induced factors in replete rats would be expected to maintain normal ENaC function. Given this logic, NaCl-restricted rats may be missing the putative maintenance factor(s), resulting in altered $\mathrm{ENaC}$ function in response to injury. Although overall immune function has not been studied in our sodium-restricted rats, malnourished rats are immunocompromised (Chandra and Dayton, 1982; Pimental and Cook, 1987; Latshaw, 1991; Hughes, 1998). If dietary $\mathrm{NaCl}$ restriction also has the effect of suppressing immune function, as does malnourishment, then there may be a lack of immune-derived factors available to sustain normal function in restricted rats. Related to this point, there is a preliminary report that dietary sodium restriction in adult rats results in less immunolabeling of immune cells in the dorsal epithelium of the tongue than in replete rats (McCluskey, 2002). Conversely, if restricted rats are not immunocompromised, a different array of immune-derived factors (e.g., different cytokines and/or growth factors) may be produced in $\mathrm{NaCl}$-restricted rats than in replete rats in response to injury that downregulate $\mathrm{ENaC}$ function. Indeed, the graded effect related to distance from intact fungiform taste buds suggests that a different set of factors controlling $\mathrm{ENaC}$ function is operational. Oddly, upregulating immune function through lipopolysaccharide injections restores normal function (Phillips and Hill, 1996), perhaps by increasing the type and/or quantity of factors released in response to injury. It is obvious that more work must be done to identify the factor(s) and their cellular/molecular mechanisms of action in regulating sodium taste responses. Regardless of the specific mechanism, there is increasing evidence that important interactions exist between gustatory and immune function.

\section{REFERENCES}

Abbas AK, Lichtman AH, Pober JS (2000) Cellular and molecular immunology, Ed 4. Philadelphia: Saunders.

Beidler LM, Smallman RL (1965) Renewal of cells within taste buds. J Cell Biol 27:263-272.

Bhave SA, Oesterle EC, Coltrera MD (1998) Macrophage and microglia-like cells in the avian inner ear. J Comp Neurol 398:241-256.

Bodeutsch N, Siebert H, Dermon C, Thanos S (1998) Unilateral injury to the adult rat optic nerve causes multiple cellular responses in the contralateral site. J Neurobiol 38:116-128.

Chandra RK, Dayton DH (1982) Trace element regulation of immunity and infection. Nutr Res 2:721-733. 
Cheal M, Oakley B (1977) Regeneration of fungiform taste buds: temporal and spatial characteristics. J Comp Neurol 172:609-626.

Cheal M, Dickey WP, Jones LB, Oakley B (1977) Taste fiber responses during reinnervation of fungiform papillae. J Comp Neurol 172:627-646.

Cleaton-Jones P (1976) A denervation study of taste buds in the soft palate of the albino rat. Arch Oral Biol 21:79-82.

Farbman AI (1980) Renewal of taste bud cells in rat circumvallate papillae. Cell Tissue Kinet 13:349-357.

Farbman AI, Hellekant G (1978) Quantitative analyses of the fiber population in rat chorda tympani nerves and fungiform papillae. Am J Anat 153:509-522.

Hellekant G (1976) The blood circulation of the tongue. Front Oral Physiol 2:130-145.

Hendricks S, Brunjes PC, Hill DL (2002) Developmental taste receptor cell kinetics. Chem Senses, in press.

Hill DL, Phillips LM (1994) Functional plasticity of regenerated and intact taste receptors in adult rats unmasked by dietary sodium restriction. J Neurosci 14:2904-2910.

Hughes DA (1998) The influence of the diet on the maturation of the immune system. Allergy [Suppl 46] 53:26-28.

Jones JE, Corwin JT (1993) Replacement of lateral line sensory organs during tail regeneration in salamanders: identification of progenitor cells and analysis of leukocyte activity. J Neurosci 13:1022-1034.

Jones JE, Corwin JT (1996) Regeneration of sensory cells after laser ablation in the lateral line system: hair cell lineage and macrophage behavior revealed by time-lapse video microscopy. J Neurosci 16:649-662.

Latshaw JD (1991) Nutrition-mechanisms of immunosuppression. Vet Immunol Immunopathol 30:111-120.

Lu X, Richardson PM (1991) Inflammation near the nerve cell body enhances axonal regeneration. J Neurosci 11:972-978.
Maher WP (1985) Arterial, venous, lymphatic pathways in dorsal mucosa of dog tongue. Microcirc Endothelium Lymphatics 2:161-184.

McCluskey LP (2002) Inflammatory cells in the normal and denervated lingual epithelium. Chem Senses, in press.

Miller Jr IJ, Spangler KM (1982) Taste bud distribution and innervation on the palate of the rat. Chem Senses 7:99-108.

Oakley B (1970) Reformation of taste buds by crossed sensory nerves in the rat's tongue. Acta Physiol Scand 79:88-94.

Phillips LM, Hill DL (1996) Novel regulation of peripheral gustatory function by the immune system. Am J Physiol 271:R857-R862.

Pimental JL, Cook ME (1987) Suppressed humoral immunity in chicks fed diets deficient in sodium, chloride, or both sodium and chloride. Poult Sci 66:2005-2010.

Popovich PG, Stokes BT, Whitacre CC (1996) Concept of autoimmunity following spinal cord injury: possible roles for $\mathrm{T}$ lymphocytes in the traumatized central nervous system. J Neurosci Res 45:349-363.

State FA (1977) Histological changes following unilateral reinnervation of the circumvallate papilla of rat. Acta Anat 98:413-419.

Suzuki Y, Takeda M, Obara N, Nagai Y (1996) Phagocytic cells in the taste buds of rat circumvallate papillae after denervation. Chem Sens 21:467-476.

Suzuki Y, Takeda M, Obara N (1997) Effect of denervation on lymphocytes and dendritic cells in the ra circumvallate and foliate papillae. Anat Embryol 196:447-455.

Warchol ME (1997) Macrophage activity in organ cultures of the avian cochlea: demonstration of a resident population and recruitment to sites of hair cell lesions. J Neurobiol 33:724-734.

Whiteside B (1926) The regeneration of the gustatory apparatus in the rat. J Comp Neurol 40:33-45.

Zalewski AA (1972) Regeneration of taste buds after transplantation of tongue and ganglia grafts to the anterior chamber of the eye. Exp Neurol 35:519-528. 